\title{
A conditional result on Goldbach numbers in short intervals
}

\author{
by
}

\section{A. Languasco (Genova)}

1. Introduction. Define a Goldbach number (G-number) to be an even number representable as a sum of two primes, and write $L=\log X$. The first result concerning the existence of G-numbers in short intervals is due to Linnik [11] who proved, assuming the Riemann Hypothesis (RH), that for any $\varepsilon>0$ and $X$ sufficiently large, the interval $\left[X, X+L^{3+\varepsilon}\right]$ contains a G-number. Linnik's result was improved by Kátai [8] and, independently, by Montgomery-Vaughan [14] who showed that the interval $\left[X, X+C L^{2}\right]$ contains a G-number for some constant $C$ and $X$ sufficiently large. Other proofs of the Kátai and Montgomery-Vaughan result have recently been obtained by Goldston [3] and Languasco-Perelli [9].

The main aim of this paper is to study the distribution of G-numbers in short intervals under the assumption of RH and Montgomery's pair correlation conjecture, a form of which asserts that

$$
F(X, T) \sim \frac{1}{2 \pi} T \log T \quad \text { for } X \rightarrow \infty
$$

uniformly for $X^{\varepsilon} \leq T \leq X$, for every fixed $\varepsilon>0$, where

$$
F(X, T)=4 \sum_{0<\gamma_{1}, \gamma_{2} \leq T} \frac{X^{i\left(\gamma_{1}-\gamma_{2}\right)}}{4+\left(\gamma_{1}-\gamma_{2}\right)^{2}}
$$

and $\gamma_{j}, j=1,2$, run over the imaginary part of the nontrivial zeros of the Riemann zeta-function $\zeta(s)$.

It is easy to see that

$$
F(X, T) \ll T \log ^{2} T
$$

uniformly in $X$. Moreover, Montgomery [13] (see also Goldston-Montgomery

1991 Mathematics Subject Classification: Primary 11P32. 
[4]) proved, again under $\mathrm{RH}$, that

$$
F(X, T) \sim \frac{1}{2 \pi} T \log X \quad \text { for } X \rightarrow \infty
$$

holds uniformly for $X \leq T$.

In the following we will denote by MC the hypothesis (1) and by WMC a weaker form of it where $\ll$ replaces $\sim$. As a slight generalization of WMC, we state the following

Hypothesis. Let $\theta \in[1,2)$. For any $\varepsilon>0$ the estimate

$$
F(X, T) \ll T(\log T)^{\theta}
$$

holds uniformly for $X^{\varepsilon} \leq T \leq X$.

We will denote the hypothesis above by $\operatorname{WMC}(\theta)$. Observe that WMC $=\mathrm{WMC}(1)$ and that, for $\theta \geq 2$, (4) is implied by (2). Our result is

Theorem. Let $\theta \in[1,2)$ be fixed and $1 /(2 X) \leq \xi \leq 1 / 2$. Assume $R H$ and $W M C(\theta)$ uniformly in the range $2 X \xi \leq T \leq X$. Then

$$
\int_{-\xi}^{\xi}\left|\sum_{X \leq n \leq 2 X}(\Lambda(n)-1) e(n \alpha)\right|^{2} d \alpha \ll X \xi L^{\theta}+\min \left(\frac{L^{2}}{\xi(\log 2 \xi)^{2}} ; X \xi L^{\theta+2}\right),
$$

where $\Lambda$ is the von Mangoldt function and $e(x)=\exp (2 \pi i x)$.

We remark that this theorem is an analogue of Theorem 3 of LanguascoPerelli [10]. As an application we can obtain the following result on the distribution of Goldbach numbers in short intervals.

Corollary. Let $\theta \in[1,2)$ and $H=C L^{\theta}$, where $C>0$ is a sufficiently large constant. Assume $R H$ and $W M C(\theta)$ uniformly in the range $X / H \leq$ $T \leq X$. Then, for all sufficiently large $X$, the interval $[X, X+H]$ contains a G-number.

We remark that our Corollary can be obtained using the method of Goldston [3].

We also recall that, under $\mathrm{RH}$ and $\mathrm{MC}$, Goldston [3] proved that the interval $[X, X+C L]$ contains a G-number and that Friedlander-Goldston [2] proved that the interval $\left[X, X+C \frac{(\log \log X)^{2}}{\log \log \log X}\right]$ contains a G-number assuming $\mathrm{RH}$ together with a strong form of $\mathrm{MC}$ and a suitable form of the ElliottHalberstam conjecture.

Our second aim is to prove the following result, which may have some independent interest, on the mean-square of the singular series of the Goldbach problem. 
Proposition. Let

$$
\mathfrak{S}(n)= \begin{cases}2 \prod_{p>2}\left(1-\frac{1}{(p-1)^{2}}\right) \prod_{\substack{p \mid n \\ p>2}}\left(\frac{p-1}{p-2}\right) & \text { if } n \text { is even } \\ 0 & \text { if } n \text { is odd. }\end{cases}
$$

Then

$$
\sum_{n \leq X} \mathfrak{S}(n)^{2}=2 X \prod_{p>2}\left(1+\frac{1}{(p-1)^{3}}\right)-\frac{1}{4} L^{2}+O\left(L^{5 / 3}\right) .
$$

The Proposition is a sharper version of Lemma 2 of Goldston [3], and its proof is based on the argument in Friedlander-Goldston [2].

Acknowledgments. We wish to thank Prof. A. Perelli for his encouragement. We also thank the referee for his positive suggestions and for the careful revision of this paper.

2. Some lemmas. Now we state two lemmas whose proofs follow the lines of Languasco-Perelli [10] (see also Heath-Brown [6]).

LEMMA 1. Writing

$$
\Sigma(X, T, v)=\sum_{0<\gamma \leq T} X^{i \gamma} e^{i v \gamma}
$$

we have

$$
F(X, T)=\int_{-\infty}^{\infty}|\Sigma(X, T, v)|^{2} e^{-2|v|} d v
$$

Lemma 2. Let $\alpha=\alpha(X)$ and $\beta=\beta(X)$ be real numbers satisfying $c \leq \alpha<\beta \leq C$ for some absolute constants $c, C>0$. Let $T>U \geq 0$. Then

$$
\int_{\alpha X}^{\beta X}\left|\sum_{0<\gamma \leq T} y^{i \gamma}\right|^{2} d y \ll X F(X, T)
$$

and

$$
\sum_{U<\gamma \leq T} X^{i \gamma} \ll T^{1 / 2}\left(\max _{U \leq u \leq T} F(X, u)\right)^{1 / 2} .
$$

The next lemma is an analogue of Lemma 2 in which we insert a factor $1 / \varrho$ in the summation on the $\gamma$.

Lemma 3. Let $\varrho=1 / 2+i \gamma, \alpha=\alpha(X)$ and $\beta=\beta(X)$ be real numbers satisfying $c \leq \alpha<\beta \leq C$ for some absolute constants $c, C>0$. Let $T>U \geq 0$. Then 


$$
\int_{\alpha X}^{\beta X}\left|\sum_{U<\gamma \leq T} \frac{y^{i \gamma}}{\varrho}\right|^{2} d y \ll X\left(\frac{F(X, T)}{T^{2}}+\frac{F(X, U)}{U^{2}}+\frac{1}{U^{1 / 2}} \int_{U}^{T} F(X, u) \frac{d u}{u^{5 / 2}}\right)
$$

and

$$
\sum_{U<\gamma \leq T} \frac{X^{i \gamma}}{\varrho} \ll\left(\frac{1}{T} \max _{U \leq u \leq T} F(X, u)\right)^{1 / 2}+\int_{U}^{T}\left(\max _{U \leq v \leq u} F(X, v)\right)^{1 / 2} \frac{d u}{u^{3 / 2}} .
$$

P roof. By partial summation

$$
\sum_{U<\gamma \leq T} \frac{X^{i \gamma}}{\varrho} \ll \frac{1}{T}\left|\sum_{U<\gamma \leq T} X^{i \gamma}\right|+\int_{U}^{T}\left|\sum_{U<\gamma \leq u} X^{i \gamma}\right| \frac{d u}{u^{2}} .
$$

The second estimate above follows immediately from the second estimate in Lemma 2. For the first estimate we have, by the Cauchy-Schwarz inequality and Lemma 2,

$$
\begin{aligned}
\int_{\alpha X}^{\beta X} \mid & \left.\sum_{U<\gamma \leq T} \frac{y^{i \gamma}}{\varrho}\right|^{2} d y \\
& \ll \frac{1}{T^{2}} \int_{\alpha X}^{\beta X}\left|\sum_{U<\gamma \leq T} y^{i \gamma}\right|^{2} d y+\int_{\alpha X}^{\beta X}\left|\int_{U}^{T}\right| \sum_{U<\gamma \leq u} y^{i \gamma}\left|\frac{d u}{u^{2}}\right|^{2} d y \\
& \ll \frac{X}{T^{2}}(F(X, T)+F(X, U))+\int_{\alpha X}^{\beta X}\left(\int_{U}^{T}\left|\sum_{U<\gamma \leq u} y^{i \gamma}\right|^{2} \frac{d u}{u^{5 / 2}}\right)\left(\int_{U}^{T} \frac{d u}{u^{3 / 2}}\right) d y \\
& \ll \frac{X}{T^{2}}(F(X, T)+F(X, U))+\frac{1}{U^{1 / 2}} \int_{U}^{T} X(F(X, u)+F(X, U)) \frac{d u}{u^{5 / 2}} \\
& \ll X\left(\frac{F(X, T)}{T^{2}}+\frac{F(X, U)}{U^{2}}+\frac{1}{U^{1 / 2}} \int_{U}^{T} F(X, u) \frac{d u}{u^{5 / 2}}\right) .
\end{aligned}
$$

3. Proof of the Theorem and of the Corollary. Writing Selberg's integral

$$
J(X, 2 X, H)=\int_{X}^{2 X}|\psi(t+H)-\psi(t)-H|^{2} d t,
$$

where $\psi(t)=\sum_{n \leq t} \Lambda(n)$, we get, by Gallagher's lemma (see, e.g., Montgomery [12], Lemma 1.9), 


$$
\begin{aligned}
& \int_{-\xi}^{\xi}\left|\sum_{X \leq n \leq 2 X}(\Lambda(n)-1) e(n \alpha)\right|^{2} d \alpha \\
& \ll \xi^{2}\left[\int_{X-1 /(2 \xi)}^{X}\left|\psi\left(t+\frac{1}{2 \xi}\right)-\psi(X)-\left(t-X+\frac{1}{2 \xi}\right)\right|^{2} d t\right. \\
& \left.\quad+J\left(X, 2 X-\frac{1}{2 \xi}, \frac{1}{2 \xi}\right)+\int_{2 X-1 /(2 \xi)}^{2 X}|\psi(2 X)-\psi(t)-(2 X-t)|^{2} d t\right] \\
& \quad+X \xi^{2}+\xi \\
& =\xi^{2}\left(I_{1}+I_{2}+I_{3}\right)+O\left(X \xi^{2}+\xi\right)
\end{aligned}
$$

say. We remark that the term $O\left(X \xi^{2}+\xi\right)$ in (5) arises from the $O(1)$ term in the estimate $\sum_{a<n<b} 1=b-a+O(1)$ applied in the above integrals.

Using the explicit formula (see Davenport [1], Ch. 17)

$$
\psi(x)=x-\sum_{|\gamma| \leq K} \frac{x^{\varrho}}{\varrho}+O\left(\frac{x(\log x K)^{2}}{K}\right)+O\left(\log x \min \left(1 ; \frac{x}{K\|x\|}\right)\right),
$$

where $\|x\|=\min _{n \in \mathbb{N}}|x-n|$, with $K=X L^{2} \xi^{1 / 2}$, we get, by (5),

$$
\int_{-\xi}^{\xi}\left|\sum_{X \leq n \leq 2 X}(\Lambda(n)-1) e(n \alpha)\right|^{2} d \alpha \ll \xi^{2}\left(J_{1}+J_{2}+J_{3}\right)+O(X \xi),
$$

where

$$
\begin{aligned}
& J_{1}=\int_{X-1 /(2 \xi)}^{X}\left|\sum_{0<\gamma \leq K} \frac{(y+1 /(2 \xi))^{\varrho}-X^{\varrho}}{\varrho}\right|^{2} d y, \\
& J_{2}=\int_{X}^{2 X-1 /(2 \xi)}\left|\sum_{0<\gamma \leq K} \frac{(y+1 /(2 \xi))^{\varrho}-y^{\varrho}}{\varrho}\right|^{2} d y, \\
& J_{3}=\int_{2 X-1 /(2 \xi)}^{2 X}\left|\sum_{0<\gamma \leq K} \frac{(2 X)^{\varrho}-y^{\varrho}}{\varrho}\right|^{2} d y .
\end{aligned}
$$

We first consider the terms in $J_{1}+J_{2}+J_{3}$ where $0<\gamma \leq 2 X \xi$, and show that they make a contribution

$$
\ll \frac{1}{X \xi^{2}} \int_{\alpha X}^{\beta X}\left|\sum_{0<\gamma \leq 2 X \xi} y^{i \gamma}\right|^{2} d y
$$


Using the Cauchy-Schwarz inequality with $0<V<W$ we get

$$
\left|\sum_{0<\gamma \leq U} \frac{W^{\varrho}-V^{\varrho}}{\varrho}\right|^{2}=\left|\int_{V}^{W} \sum_{0<\gamma \leq U} u^{\varrho-1} d u\right|^{2} \leq|W-V| \int_{V}^{W}\left|\sum_{0<\gamma \leq U} u^{i \gamma}\right|^{2} \frac{d u}{u} .
$$

Applying this estimate in $J_{1}, J_{2}$ and $J_{3}$ we obtain (7). By Lemma 2 and $\operatorname{WMC}(\theta)$ the right hand side of $(7)$ is $\ll F(X, 2 X \xi) / \xi^{2} \ll X L^{\theta} / \xi$, which, by (6), makes the contribution $X \xi L^{\theta}$.

Now we consider the contribution from the terms $2 X \xi<\gamma \leq K$ in $J_{1}+J_{2}+J_{3}$. We see immediately that this contribution is

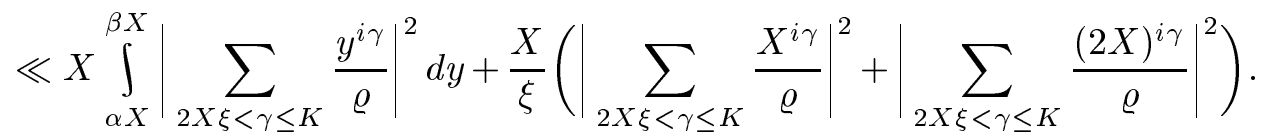

By Lemma 3 and $\operatorname{WMC}(\theta)$ the first term is $\ll X L^{\theta} / \xi$. The other terms on the right come from $J_{1}$ and $J_{3}$. By Lemma 3 and $\operatorname{WMC}(\theta)$ they contribute $\ll X L^{2+\theta} / \xi$; alternatively they are bounded by $I_{1}+I_{3}$, which by the Brun-Titchmarsh theorem is $\ll L^{2} /\left(\xi^{3}(\log 2 \xi)^{2}\right)$. These estimates, combined with (6), give the second error term in the Theorem.

Now we prove the Corollary. Let $H=\left[C L^{\theta}\right]$, where $C \geq 1$ is a constant. Define

$$
L(\alpha)=\left|\sum_{m=1}^{H} e(-m \alpha)\right|^{2}=\sum_{m=-H}^{H} a(m) e(-m \alpha),
$$

where $a(m)=H-|m|$,

$$
\begin{gathered}
R(n)=\sum_{h+k=n} \Lambda(h) \Lambda(k), \quad S(\alpha)=\sum_{X \leq n \leq 2 X} \Lambda(n) e(n \alpha), \\
T(\alpha)=\sum_{X \leq n \leq 2 X} e(n \alpha), \quad E(\alpha)=S(\alpha)^{2}-T(\alpha)^{2} .
\end{gathered}
$$

We have

$$
\begin{aligned}
\sum_{n=X-H}^{X+H} a(n-X) R(n)= & \int_{-1 / 2}^{1 / 2} S(\alpha)^{2} L(\alpha) e(-X \alpha) d \alpha \\
= & \int_{-1 / 2}^{1 / 2} T(\alpha)^{2} L(\alpha) e(-X \alpha) d \alpha \\
& +\int_{-1 / 2}^{1 / 2} E(\alpha) L(\alpha) e(-X \alpha) d \alpha=A+B
\end{aligned}
$$


say. It is easy to prove that

$$
A=\sum_{n=X-H}^{X+H} a(n-X) \sum_{h+k=n} 1=H^{2} X+O\left(H^{3}\right) .
$$

Now we proceed to estimate $B$. Using

$$
T(\alpha) \ll \min (X ; 1 /|\alpha|) \quad \text { for }|\alpha| \leq 1 / 2
$$

we get

$$
\int_{-\xi}^{\xi}|T(\alpha)|^{2} d \alpha \begin{cases}\ll X^{2} \xi & \text { if } 0<\xi<1 / X \\ =X+O(1 / \xi) & \text { if } 1 / X \leq \xi \leq 1 / 2\end{cases}
$$

Hence, using the identity

$$
f^{2}-g^{2}=2 f(f-g)-(f-g)^{2},
$$

the Cauchy-Schwarz inequality and (10), we have

$$
\int_{-\xi}^{\xi}|E(\alpha)| d \alpha \ll\left(X \int_{-\xi}^{\xi}|S(\alpha)-T(\alpha)|^{2} d \alpha\right)^{1 / 2}+\int_{-\xi}^{\xi}|S(\alpha)-T(\alpha)|^{2} d \alpha
$$

provided $1 / X \leq \xi \leq 1 / 2$.

Since

$$
L(\alpha) \ll \min \left(H^{2} ; 1 /|\alpha|^{2}\right) \quad \text { for }|\alpha| \leq 1 / 2,
$$

we have

$$
B \ll H^{2} \int_{-1 / H}^{1 / H}|E(\alpha)| d \alpha+\int_{1 / H}^{1 / 2}|E(\alpha)| \frac{d \alpha}{\alpha^{2}} .
$$

From the Theorem and (11) we get

$$
H^{2} \int_{-1 / H}^{1 / H}|E(\alpha)| d \alpha \ll H^{3 / 2} X L^{\theta / 2} .
$$

By partial integration, the Theorem and (11) we obtain

$$
\int_{1 / H}^{1 / 2}|E(\alpha)| \frac{d \alpha}{\alpha^{2}} \ll H^{3 / 2} X L^{\theta / 2} .
$$

Hence from (13)-(15) we have

$$
B \ll H^{3 / 2} X L^{\theta / 2},
$$

and from (8), (9) and (16) we get 


$$
\sum_{n=X-H}^{X+H} a(n-X) R(n) \gg H^{2} X
$$

provided that $C$ is sufficiently large. Thus the Corollary follows.

4. Proof of the Proposition. Let

$$
\mathfrak{S}=\prod_{p>2}\left(1-\frac{1}{(p-1)^{2}}\right)
$$

We have

$$
\begin{aligned}
\sum_{n \leq X} \mathfrak{S}(n)^{2} & =4 \mathfrak{S}^{2} \sum_{2 n \leq X} \prod_{\substack{p \mid n \\
p>2}}\left(\frac{p-1}{p-2}\right)^{2} \\
& =4 \mathfrak{S}^{2} \sum_{2 n \leq X} \prod_{\substack{p \mid n \\
p>2}}\left(1+\frac{2 p-3}{(p-2)^{2}}\right) \\
& =4 \mathfrak{S}^{2} \sum_{n \leq X / 2} \sum_{j \mid n} f(j),
\end{aligned}
$$

where

$$
f(j)= \begin{cases}\mu^{2}(j) \prod_{p \mid j} \frac{2 p-3}{(p-2)^{2}} & \text { if } j \text { is odd } \\ 0 & \text { if } j \text { is even. }\end{cases}
$$

Then, changing the order of summation in (18), we obtain

$$
\begin{aligned}
\sum_{n \leq X} \mathfrak{S}(n)^{2}= & 4 \mathfrak{S}^{2} \sum_{j \leq X / 2} f(j)\left[\frac{X}{2 j}\right] \\
= & 2 \mathfrak{S}^{2} X \sum_{j=1}^{\infty} \frac{f(j)}{j}-2 \mathfrak{S}^{2} X \sum_{j>X / 2} \frac{f(j)}{j} \\
& -2 \mathfrak{S}^{2} \sum_{j \leq X / 2} f(j)-4 \mathfrak{S}^{2} \sum_{j \leq X / 2} f(j) P\left(\frac{X}{2 j}\right),
\end{aligned}
$$

where $P(u)=u-[u]-1 / 2$.

By straightforward computations we get

$$
\begin{aligned}
2 \mathfrak{S}^{2} \sum_{j=1}^{\infty} \frac{f(j)}{j} & =2 \prod_{p>2}\left(1-\frac{1}{(p-1)^{2}}\right)^{2}\left(1+\frac{2 p-3}{p(p-2)^{2}}\right) \\
& =2 \prod_{p>2}\left(1+\frac{1}{(p-1)^{3}}\right) .
\end{aligned}
$$


Next, we will show

$$
\sum_{j \leq U} f(j)=\frac{1}{8 \mathfrak{S}^{2}} \log ^{2} U+B \log U+O(1),
$$

where $B$ is a constant. By partial summation this implies

$$
\sum_{j>U} \frac{f(j)}{j}=\frac{1}{4 \mathfrak{S}^{2}} \cdot \frac{\log U}{U}+O\left(\frac{1}{U}\right)
$$

The Proposition follows from (19)-(22) together with the estimate

$$
\sum_{j \leq X} f(j) P(X / j) \ll L^{5 / 3}
$$

Now we prove (21). Writing

$$
H(s)=\sum_{m=1}^{\infty} f(m) m^{-s}=\prod_{p>2}\left(1+\frac{2 p-3}{p^{s}(p-2)^{2}}\right)
$$

we see that $H(s)$ is an analytic function for $\operatorname{Re} s=\sigma>0$ and, using the Perron formula with error term (see, e.g., Lemma 3.12 of Titchmarsh [15]), we obtain

$$
\sum_{j \leq U} f(j)=\frac{1}{2 \pi i} \int_{c-i Z}^{c+i Z} H(s) \frac{U^{s}}{s} d s+O\left(U^{c} \sum_{m=1}^{\infty} \frac{|f(m)|}{m^{c}(1+Z|\log (U / m)|)}\right),
$$

where $\varepsilon>0$ is a fixed constant, $c=\varepsilon+1 / \log U<1 / 4$ and $Z$ will be chosen later on.

The error term can be estimated using

$$
f(m) \ll \frac{d(m)}{m} \sum_{h \mid m} \frac{d^{2}(h)}{h}
$$

(see (30) of Goldston [3]), where $d(m)$ is the divisor function, and the classical estimates $\sum_{v \leq m} d(v)^{q} \ll m(\log m)^{2^{q}-1}$ (see, e.g., Theorem 5.3 of Hua [7]) and $d(m) \ll m^{\varepsilon}$ (see, e.g., Theorem 315 of Hardy-Wright [5]). So we have

$$
\sum_{j \leq U} f(j)=\frac{1}{2 \pi i} \int_{c-i Z}^{c+i Z} H(s) \frac{U^{s}}{s} d s+O\left(\frac{U^{c}}{Z}\right) .
$$

Now we observe that

$$
H(s)=\left(1-\frac{1}{2^{s+1}}\right)^{2} \zeta(s+1)^{2} g(s)
$$

where

$$
g(s)=\prod_{p>2}\left(1-\frac{1}{p^{s+1}}\right)^{2}\left(1+\frac{2 p-3}{p^{s}(p-2)^{2}}\right)
$$


which converges absolutely and is analytic for $\sigma>-1 / 2$. So $H(s) U^{s} / s$ has a triple pole at $s=0$ with residue

$$
\frac{1}{8 \mathfrak{S}^{2}} \log ^{2} U+B \log U+O(1)
$$

Consider a rectangular contour with right side $s=c+i t, t \in[-Z, Z]$, and left side $-1 / 4+i t, t \in[-Z, Z]$. The contribution of the top, bottom and left sides of the contour can be estimated using $\zeta(\sigma+i t) \ll t^{1 / 6}$ for $\sigma \geq 1 / 2$ (see, e.g., Titchmarsh [15], p. 115). Hence we have

$$
\sum_{j \leq U} f(j)=\frac{1}{8 \mathfrak{S}^{2}} \log ^{2} U+B \log U+O\left(Z^{-2 / 3} U^{c}+Z^{1 / 3} U^{-1 / 4}\right)+O(1) .
$$

Choosing $Z=U^{3 c}$, we obtain (21).

Now we prove (23). For $j$ odd we have

$$
\begin{aligned}
f(j) & =\mu^{2}(j) \prod_{p \mid j}\left(\frac{2}{p-2}\right)\left(\frac{p-3 / 2}{p-2}\right)=\frac{\mu^{2}(j) d(j)}{\varphi_{2}(j)} \prod_{p \mid j}\left(1+\frac{1}{2(p-2)}\right) \\
& =\frac{\mu^{2}(j) d(j)}{\varphi_{2}(j)} \sum_{\delta \mid j} \frac{\mu^{2}(\delta)}{2^{\omega(\delta)} \varphi_{2}(\delta)},
\end{aligned}
$$

where $\omega(n)$ is the number of distinct prime factors of $n, \varphi_{2}(p)=p-2$ and $\varphi_{2}$ is extended to square-free numbers by multiplicativity.

Hence, interchanging the order of summation, we obtain

$$
\sum_{j \leq X} f(j) P\left(\frac{X}{j}\right)=\sum_{\substack{\delta \leq X \\(\delta, 2)=1}} \frac{\mu^{2}(\delta) d(\delta)}{2^{\omega(\delta)}\left(\varphi_{2}(\delta)\right)^{2}}\left(\sum_{\substack{k \leq X / \delta \\(k, 2)=1}} \frac{\mu^{2}(k) d(k)}{\varphi_{2}(k)} P\left(\frac{X / \delta}{k}\right)\right) .
$$

Using the argument in (2.9)-(2.13) of Friedlander-Goldston [2], we find that the inner sum can be estimated by

$$
\sum_{n \leq X} \frac{d(n)}{n} P(X / n)
$$

which is $\ll L^{5 / 3}$ by the remark at the end of Section 2 of [2]. Using this estimate we obtain

$$
\sum_{j \leq X} f(j) P\left(\frac{X}{j}\right) \ll L^{5 / 3} \sum_{\substack{\delta \leq X \\(\delta, 2)=1}} \frac{\mu^{2}(\delta) d(\delta)}{\left(\varphi_{2}(\delta)\right)^{2}}
$$

and hence (23) follows from the convergence of the series

$$
\sum_{\substack{\delta=1 \\(\delta, 2)=1}}^{\infty} \frac{\mu^{2}(\delta) d(\delta)}{\left(\varphi_{2}(\delta)\right)^{2}}
$$




\section{References}

[1] H. Daven port, Multiplicative Number Theory, 2nd ed., Springer, 1980.

[2] J. B. Friedlander and D. A. Goldston, Some singular series averages and the distribution of Goldbach numbers in short intervals, Illinois J. Math. 39 (1995), $158-180$.

[3] D. A. Goldston, Linnik's theorem on Goldbach numbers in short intervals, Glasgow Math. J. 32 (1990), 285-297.

[4] D. A. Goldston and H. L. Montgomery, Pair correlation of zeros and primes in short intervals, in: Analytic Number Theory and Dioph. Probl., A. C. Adolphson et al. (eds.), Birkhäuser, 1987, 183-203.

[5] G. H. Hardy and E. M. Wright, An Introduction to the Theory of Numbers, 5th ed., Clarendon Press, 1990.

[6] D. R. Heath-Brown, Gaps between primes, and the pair correlation of zeros of the zeta-function, Acta Arith. 41 (1982), 85-99.

[7] L. K. Hua, Introduction to Number Theory, Springer, 1982.

[8] I. Kátai, A remark on a paper of Yu. V. Linnik, Magyar Tud. Akad. Mat. Fiz. Oszt. Közl. 17 (1967), 99-100 (in Hungarian).

[9] A. Languasco and A. Perelli, On Linnik's theorem on Goldbach numbers in short intervals and related problems, Ann. Inst. Fourier (Grenoble) 44 (1994), 307-322.

[10] - - - A pair correlation hypothesis and the exceptional set in Goldbach's problem, Mathematika 43 (1996), 349-361.

[11] Yu. V. Linnik, Some conditional theorems concerning the binary Goldbach problem, Izv. Akad. Nauk SSSR Ser. Mat. 16 (1952), 503-520 (in Russian).

[12] H. L. Montgomery, Topics in Multiplicative Number Theory, Lecture Notes in Math. 227, Springer, 1971.

[13] - The pair correlation of zeros of the zeta function, in: Proc. Sympos. Pure Math. 24, Amer. Math. Soc., 1973, 181-193.

[14] H. L. Montgomery and R. C. Vaughan, The exceptional set in Goldbach's problem, Acta Arith. 27 (1975), 353-370.

[15] E. C. Titchmarsh, The Theory of Riemann Zeta-Function, 2nd ed., Oxford Univ. Press, 1986.

Dipartimento di Matematica

Università di Genova

Via Dodecaneso 35

16146 Genova, Italy

E-mail: languasco@dima.unige.it

Received on 30.4.1996

and in revised form on 8.9.1997 